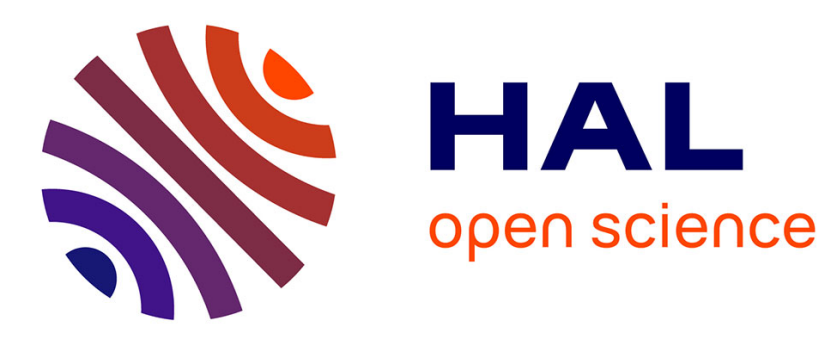

\title{
Pédagogie muséale et enseignement du volcanisme à la Réunion
}

\author{
Azzedine Si Moussa, Nadine Deurveilher
}

\section{To cite this version:}

Azzedine Si Moussa, Nadine Deurveilher. Pédagogie muséale et enseignement du volcanisme à la Réunion. Carrefours de l'éducation, 2003, 16, pp.57-71. halshs-00004910

\section{HAL Id: halshs-00004910 \\ https://shs.hal.science/halshs-00004910}

Submitted on 10 Oct 2005

HAL is a multi-disciplinary open access archive for the deposit and dissemination of scientific research documents, whether they are published or not. The documents may come from teaching and research institutions in France or abroad, or from public or private research centers.
L'archive ouverte pluridisciplinaire HAL, est destinée au dépôt et à la diffusion de documents scientifiques de niveau recherche, publiés ou non, émanant des établissements d'enseignement et de recherche français ou étrangers, des laboratoires publics ou privés. 


\title{
Pédagogie muséale et enseignement du volcanisme à la Réunion
}

\author{
A Azzedine Si Moussa \\ université de la Réunion, chercheur associé à l'IREDU-CNRS. \\ $\triangle$ Nadine Deurveilher \\ Stella Matutina.
}

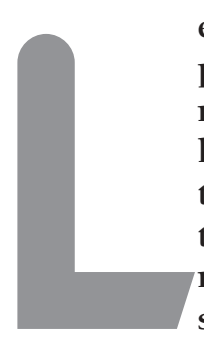

es recherches sur la pédagogie muséale mettent en évidence la double transposition des principes théoriques de la médiatique des sciences et de la psycho-pédagogie. L'enjeu est de comprendre pourquoi et comment les élèves peuvent construire leurs apprentissages scientifiques en milieu muséal. La présente contribution propose une réflexion sur cette problématique à partir de l'exemple de l'enseignement du volcanisme à l'île de la Réunion. L'analyse d'entretiens menés avec des professeurs des écoles, de collèges et de lycées montre dans quelle mesure les élèves bénéficient d'un impact pédagogique significatif dans le cadre des visites effec- tuées dans un musée dédié au Piton de la Fournaise. Les résultats montrent que les enseignants, au-delà d'objectifs d'acquisitions de connaissances simples, mettent en évidence des apports non négligeables au plan de la communication et de la socialisation. Mais la perception d'un enseignement de la géologie relativement complexe à mettre en ouvre questionne la place de la pédagogie muséale, en tant que complément ou support décisif, au regard des représentations des attentes des professeurs et des aptitudes des élèves. 


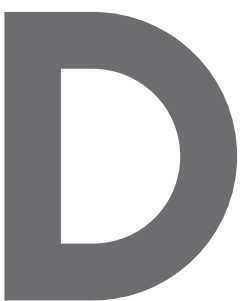

epuis plusieurs décennies, les enseignants sont amenés à « ouvrir l'école vers l'extérieur » en organisant les visites extrascolaires. En France, le ministère de l'Éducation nationale et de la Culture a créé en 1982 la mission Musée afin de dynamiser les musées de province, créer de nouveaux établissements et veiller au développement d'un réseau national de structures locales regroupant les centres de culture scientifique et technique, et améliorer les rapports entre ces structures muséales et le monde scolaire ${ }^{1}$. Selon Guichard et Martinand (1999), les musées et les expositions scientifiques et techniques cherchent de plus en plus à tirer leur légitimité d'un objectif éducatif; d'autre part, le système éducatif éprouve le besoin de s'ouvrir vers l'extérieur et en particulier d'utiliser les expositions comme des ressources pédagogiques.

\section{Le développement du lien musée-école}

Nous proposons ici une contribution sur une partie des enjeux pédagogiques de cette rencontre école musée. Il s'agit plus particulièrement d'analyser comment les enseignants prennent l'initiative de recourir à ce qu'il est convenu d'appeler la «pédagogie muséale » et dans quelle mesure on peut identifier les conditions de son efficacité pédagogique. Dans cette perspective, il serait logique de s'intéresser aux nombreux aspects de la relation école musée. On pense notamment aux différentes conceptions visant à évaluer cette relation, par exemple en termes de « déscolarisation» ou «parascolarisation» du musée (Paquin, 1998), ou à l'inverse montrant une excessive «scolarisation » de l'espace muséal (Cohen, 2000; Van Praët et Poucet, 1992). De même, il conviendrait de rappeler la construction de modèles théoriques d'utilisation du musée dans un but pédagogique (Allard et Boucher, 1991; 1998; Larouche, 1999).

Notre objectif n'est toutefois pas de procéder à une vaste revue de la littérature, très riche en ce domaine, mais de privilégier des développements consacrés à notre expérimentation sur le terrain, rendue originale par son contexte in situ, c'est-à-dire en prise directe avec un site volcanique ${ }^{2}$. C'est pourquoi nous nous limiterons à une présentation relativement synthétique et nécessairement partielle de quelques exemples de travaux illustrant le développement des recherches sur l'école et le musée.

1. En décembre 2000, un plan pour le développement des arts et de la culture à l'école prévoyait que «tout établissement culturel ou artistique subventionné sera doté d'un service éducatif chargé de concevoir et de mettre en œuvre des actions avec des partenaires éducatifs » (Héritier-Augé, 1991).

2. Il s'agit du Piton de la Fournaise, un des plus grands volcans actifs du monde (7000 mètres d'altitude, dont 3000 émergés). 
Nous verrons dans un premier temps que l'émergence de la pédagogie muséale, ou si l'on préfère des activités d'apprentissage en milieu muséal, résulte, sur le plan théorique, d'une double transposition des principes issus de travaux menés en médiatique des sciences et des apports de diverses recherches dans le domaine de la psychopédagogie.

Nous montrerons ensuite, en prenant pour exemple l'enseignement du volcanisme, ce qu'il advient de cette transposition au plan des pratiques pédagogiques des enseignants. Nous questionnerons ainsi, à travers leurs réflexions dans ce domaine, l'impact pédagogique qu'offre le recours à des ressources extérieures à l'école, au collège ou au lycée, ressources qui sont donc représentées ici par la Maison du Volcan de l'Ile de la Réunion.

\section{Médiatique des sciences et apprentissages scolaires}

L'analyse médiatique des visites dans le domaine des expositions scientifiques constitue un point de départ incontournable dans une réflexion préalable sur l'émergence d'une pédagogie muséale ou plus simplement d'une démarche d'apprentissage en milieu muséal. Le recours aux principes et concepts des constructions théoriques de la didactique des sciences et de la psychologie contribue par la suite à la constitution d'un cadre théorique d'expérimentation pédagogique.

L'approche sociopolitique prônée par Bernard Schiele montre par exemple qu'il existe certes des éléments qui collaborent à l'émergence du sens pour le visiteur de l'exposition, mais le message transmis n'est qu'un point de départ et le sens reste à construire par le visiteur: «l'interprétation du sens de l'exposition s'ancre dans la signifiance des pratiques sociales » (Schiele \& Koster, 1998). Autrement dit, le visiteur interprète ou donne un sens aux choses qu'il voit à travers son propre cadre de référence, c'est-à-dire à travers son schème de pensée. À l'opposé, l'approche sociosémiotique de Davallon (in Guichard et Martinand, 1999; p. 63-65) cherche à décrire le système de l'exposition à partir des « caractéristiques internes qui la constituent». Il considère que la production de sens résulte des opérations de mise en exposition.

La recherche des facteurs de causalité dans la production de sens a conduit à s'interroger sur le rôle de l'organisation de la visite: le contexte de son déroulement, le temps que le visiteur y consacre, le fait qu'elle soit une activité sociale et de groupe, les motifs de visite... Toutefois, selon Giordan (1998), certains acquis dans le domaine pédagogique, comme ceux concernant les conceptions des apprenants «ne dépendent pas du lieu et semblent être transposables» à la pédagogie informelle dans le cadre d'une visite d'exposition. Les pratiques de référence des apprenants sont essentielles dans les expositions où les objets et les mises en situation sont les moyens de communication: c'est « au travers d'elles que l'apprenant interprète de nouvelles informations » (De Vecchi \& Giordan, 1996).

Le rôle prépondérant des objets et des mises en situation rapproche la pédago- 
gie muséale des pédagogies actives. Elle s'appuie alors sur l'émotion (notion de plaisir) et l'implication (invitation à toucher grâce à l'existence des dispositifs interactifs). Les méthodes d'étude de la psychologie de la cognition poussent les chercheurs à analyser les mécanismes cognitifs des visiteurs d'exposition en empruntant les concepts de la didactique comme les niveaux de formulation et les trames conceptuelles.

L'interactivité du sujet avec les objets d'exposition permet d'apprendre par l'apprentissage par essai-erreur: le visiteur est mis en situation de tester ses hypothèses et d'établir un lien de causalité avec le résultat de son action qu'il peut modifier jusqu'à obtenir le résultat souhaité. Cet aspect de l'interactivité se rapproche de la démarche expérimentale.

L'interactivité donne à la fois un statut positif à la découverte, et à la fois confrontation aux ignorances, stimule le questionnement et l'accès à des savoirs ponctuels mais fonctionnels. Les manipulations d'expositions sont des dispositifs matériels, manipulables par les visiteurs, permettant une compréhension immédiate de l'objectif à atteindre. Elles représentent en trois dimensions les phénomènes, les concepts, les procédés et les démarches de la recherche (Billet, 1996).

Une autre forme d'interactivité correspond à des moyens d'accéder à des informations, de manipuler des banques de données et d'explorer les contenus: «L'enfant observe, écoute, fait un effort afin de percevoir et comprendre (Jantzen, 1995).

Dans une exposition, les enfants apprennent aussi par interaction entre eux ou avec les adultes qui les accompagnent (Bruner, 1983). Cette collaboration avec les adultes, ou relation d'étayage, consiste à protéger l'enfant contre les distractions en assurant une convergence entre son attention et celle de l'adulte, à donner à l'enfant l'occasion d'établir des relations entre signes et événements, limiter la tâche à un niveau accessible à l'enfant, fournir des moyens pour la représentation et l'exécution des relations entre moyens et but, permettre à l'adulte de s'accorder au niveau de l'enfant dans le déroulement de la tâche. Bruner montre aussi l'importance du contexte psychologique pour l'apprentissage. Le fait d'éprouver du plaisir fait partie de ce contexte. Le plaisir est lié d'abord, chez l'enfant, aux perceptions sensorielles. Les situations ludiques permettent également de découvrir des process scientifiques ou techniques. Le jeu procure du plaisir par la manipulation des objets et l'émotion des scénographies. C'est aussi un moyen de maintenir l'intérêt, et de faciliter le cheminement vers le savoir (Caillet et Coppey, 1992).

Mais l'acquisition des connaissances et le maintien de l'intérêt ne sont pas les mêmes selon que l'interactivité se réduit au presse-bouton ou qu'elle facilite un dialogue. Les manipulations utilisent de nombreux dispositifs techniques qui vont du presse-bouton (déclenche un processus) au jeu informatique (possibilité de fixer des valeurs à des paramètres et d'observer les résultats dans une logique questions-réponses).

Or explorer un phénomène consiste à faire varier les paramètres du phénomène pour faciliter la compréhension de la relation de cause à effet. Dans cette logique, 
la manipulation met en scène certains de ces paramètres, et le public, par l'action sur leurs valeurs, est interpellé par les effets spectaculaires, esthétiques, surprenants, non attendus. Ces effets sont conçus pour créer un conflit cognitif par un décalage entre ce que le savoir commun s'attend de voir apparaître et ce qui apparaît. Surpris et intéressé, le public se pose des questions dont les réponses naissent de l'interaction avec le dispositif pour atteindre un premier équilibre cognitif. Selon Piaget (1971), le savoir commun (système relativement formé de représentation et d'interprétation du monde constitué essentiellement de croyances ou de connaissances préscientifiques) est très éloigné des savoirs scientifiques et constitue très souvent un obstacle à l'acquisition de ces savoirs.

Pour déclencher un changement des représentations du public, il faut l'amener à accepter et à prendre conscience de la contradiction (conflit) avec ses opinions pour favoriser l'abandon de ses propres théories. L'interactivité est donc génératrice de conflit cognitif, stratégie permettant l'acquisition de connaissances. L'apprentissage est aussi le résultat de la coordination et de la résolution des conflits cognitifs entre les individus: «Les conflits entre centrations cognitives qui coexistent au sein d'un groupe constituent un facteur de développement cognitif plus puissant qu'un conflit entre centrations individuelles» (Doise et Mugny, 1981).

\section{La recherche des conditions d'un impact pédagogique}

L'une des premières conditions est l'élaboration par l'enseignant d'un projet pédagogique. Une étude de Pierre Clément montre que les impacts les plus ambitieux des concepteurs de l'exposition sur la carte d'identité à la Cité de la Villette ne sont totalement atteints que lors des visites préparées par l'enseignant, avec une sensibilisation et une phase de structuration et de réutilisation de découvertes dans une nouvelle situation après la visite. L'incorporation de la visite de l'exposition dans un projet pédagogique modifie le rapport au savoir. Et le contrat didactique implicite qui lie les élèves à leur enseignant lors de la visite, même si cet enseignant n'encadre pas étroitement les enfants pendant la visite, semble d'une importance capitale (Billet, 1996).

À la Maison du Volcan (que nous analyserons plus précisément par la suite), des entretiens et observations exploratoires nous ont révélé que le service pédagogique élabore des questionnaires et des fiches d'enquêtes à l'attention des élèves afin de les guider, dans le cadre d'un projet pédagogique avec l'enseignant. Or il semblerait que les élèves qui arrivent dans l'exposition sans disposer de ces outils survolent rapidement les panneaux et les maquettes, au gré de l'intérêt qu'ils portent aux objets ou aux textes. Ils regardent, s'émerveillent sans se poser de questions et lancent des exclamations d'étonnement «Ohh!... tu as vu le volcan qui gonfle! En revanche, les élèves munis d'un questionnaire se dirigent directement vers les panneaux, maquettes ou bornes vidéos qui y sont indiquées. Ils ne perdent pas de temps et lisent ou écoutent pour fournir de bonnes réponses. 
Toutefois, selon P. Floud (in Girault, 2000), au lieu d'inciter les enfants à réfléchir à des questions ouvertes qui développent leur esprit critique, favorisent une réelle observation et débouchent sur un questionnement personnel leur faisant prendre conscience de la valeur polysémique des objets muséaux, les questionnaires induisent souvent un comportement de course à la bonne réponse sans que les élèves ne tentent de comprendre globalement la trame narrative de ce qui leur est présenté.

Une autre des conditions d'un impact éducatif est de provoquer des échanges et la verbalisation. L'interaction avec l'objet a pour objectif d'amener l'élève à réfléchir, à se poser des questions. Mais on a noté que les élèves se contentaient souvent de regarder le résultat de leur action sans vraiment chercher à analyser les causes de ce résultat. C'est l'intervention de l'adulte qui, en les interpellant par des questions ou en essayant d'expliquer ce qui se passe, permet à l'élève de comprendre.

À la Maison du Volcan, lorsqu'on appuie sur un bouton, on provoque des phénomènes un volcan qui gonfle, la subduction des plaques lithosphériques... On peut aussi provoquer des séismes en sautillant devant une borne interactive. Nous avons observé des élèves faire ces simulations, mais la plupart se contentent de regarder ce qui se passe sans chercher à comprendre. C'est lorsqu'un adulte (professeur, médiateur...) l'interpelle qu'il essaie de réfléchir et d'expliquer ce qui se passe. Cela lui permet de confronter ce qu'il dit avec ce que l'adulte sait.

La question de l'encadrement pédagogique des élèves en milieu muséal semble donc centrale. C'est pourquoi nous avons privilégié une analyse du discours des enseignants susceptibles de recourir à la pédagogie muséale. Notre corpus se compose donc de professeurs ${ }^{3}$ des écoles, de collège et de lycée ayant visité, avec leurs élèves, la Maison du Volcan. Le postulat est ici que les enseignants:

- sont à même d'évaluer la pertinence de l'enseignement du volcanisme en milieu muséal (ils en prennent en tout cas l'initiative en fonction de divers paramètres que nous tenterons d'identifier);

- sont également en mesure d'évaluer l'efficacité pédagogique de leur initiative, en fonction de leur perception des acquisitions des élèves ${ }^{4}$.

D’un point de vue méthodologique, il s'agit donc d'une évaluation interne. Nous avons ainsi mené 18 entretiens semi-directifs. Tous les enseignants contactés ont manifesté un réel intérêt pour notre objet de recherche et se sont révélés dans l'ensemble relativement prolixes.

3. Les professeurs interviewés (en février-mars 2002) enseignent dans divers établissements de l'île: sept d'entre eux (El à E7) exercent en CMl et/ou CM2, cinq (C8 à C12) ont une classe de 4e et six (L13 à L18) ont une classe de $1^{\text {re }} \mathrm{S}$.

4. Notons que si notre propos est bien centré sur les acteurs professeurs et élèves, notre objectif n'est pas de dresser une typologie des uns et des autres, comme cela a pu être mené par ailleurs (voir par exemple Quagliozzi et Cohen, 2000). 


\section{Analyse des entretiens menés avec des professeurs d'école, de collège et de lycée}

Une analyse thématique de ces entretiens nous a conduit à distinguer dans un premier temps ce qui a trait à la valorisation des supports pédagogiques muséaux. Au-delà de ces aspects matériels, les enseignants interviewés ont également apporté une réflexion critique sur le problème de l'encadrement des élèves. Enfin, l'évaluation de l'impact pédagogique des activités menées en milieu muséal est appréhendée au plan des compétences comportementales (socialisation, autonomie, démarches actives...) et au niveau de l'acquisition des connaissances proprement dites (notions, compétences transversales...).

\section{Valorisation des supports pédagogiques muséaux}

La pertinence pédagogique des supports muséaux est reconnue par de nombreux enseignants. Certains, comme C11, apprécient fortement les outils mis à disposition. Chez les professeurs de lycée, ce sont les potentialités en matière de documentation et de présentations (films, maquettes, cimaises) qui constituent un élément particulièrement intéressant. De façon générale, l'objectif est bien de favoriser une certaine forme d'interactivité, même si ce terme n'est pas explicitement évoqué : il s'agit ici d'accéder à des informations, de manipuler des banques de données, d'explorer des contenus.

E4: «Il y a tout l'apprentissage scientifique, ils font chaque année l'expérience du geyser, de la caldeira, des projections. On voit un petit peu les mouvements des plaques. Ce qu'il y a aussi de très bien, c'est cette carte en relief. Les enfants apprécient beaucoup l'exposition permanente, ses cimaises, avec les petits ordinateurs. Ce qui est bien aussi, c'est l'espace ludique parce qu'on peut se regrouper. Les élèves aiment aussi les maquettes parce qu'elles ont très bien représenté et même si ce sont des enfants jeunes, ils arrivent à s'imaginer, à repérer les différents sites. »

C11: «Il y a une démarche qu'on voit bien. Il y a une liaison avec la tectonique et ça va jusqu'à la colonisation des laves par le milieu naturel, par les plantes. Les maquettes sont très attractives. Il y a une maquette étonnante, celle des chambres magmatiques. »

L15: «Le contenu peut être accessible à tous les niveaux. Les cimaises sont claires et les maquettes attractives. Ca permet de compléter les informations.»

Le jugement des enseignants n'est pas totalement positif. El remarque le manque d'expériences ou de maquettes que les élèves pourraient manipuler eux-mêmes. D'autres enseignants, comme L13, distinguent des points positifs (l'accès à la documentation) et négatifs (l'absence de renouvellement des films et des maquettes). 
L13: «Je me demande si on ne pourrait pas faire un espace plus animé, par exemple avec des expériences, des maquettes...»

L14: «C'est cette partie purement géologique de l'île qui manque. Ce qui manque également, c'est l'histoire qui peut remonter avec la tectonique des plaques, qui explique les points chauds, la formation de l'île, etc. Il manque des cartes et des photographies de la Réunion, un peu d'animations. Le problème c'est d'essayer de concurrencer la télévision en rendant plus vivants les phénomènes. Une maquette qui me plaît beaucoup... est celle des chambres magmatiques. Mais elle est inerte!»

Malgré ces réserves, le discours des professeurs illustre bien une double préoccupation, concernant d'une part l'intérêt scientifique et à visée cognitive des supports proposés, et d'autre part le caractère vivant, attractif, imaginaire de ces supports. La référence à la construction d'un savoir scientifique en rendant l'élève acteur de ses apprentissages apparaît sans ambiguité, en écho aux tendances pédagogiques actuelles.

\section{L'encadrement pédagogique}

Dans ce domaine, les enseignants ont exprimé leur opinion concernant les interventions effectuées par le personnel du service pédagogique de la Maison du Volcan. La question de la délégation de leurs prérogatives aux intervenants ne se pose pas de manière explicite: la conviction de la nécessité de cette démarche est partagée.

C'est donc essentiellement la forme des interventions pratiquées qui soulève des interrogations. Par exemple, E6 formule une appréciation très positive de l'intervention mais la juge «trop abstraite » pour les enfants (sur le thème des roches volcaniques). De son côté, E2 estime qu'il y a trop d'informations et que c'est trop compliqué pour des élèves. De même, trois enseignants de collège ( $\mathrm{C} 8, \mathrm{C} 10$ et C11) dressent un bilan sans complaisance de la qualité et de l'apport des interventions effectuées.

E2: «Le contenu était trop compliqué pour de jeunes enfants. Ils sont noyés sous les informations. D'ailleurs, au bout de trois quarts d'heure les élèves ont commencé à s'ennuyer et ils me disaient: "Monsieur, quand est-ce qu'on part?" Je leur avais fait un petit questionnaire qu'ils ont rempli un peu au hasard, en recopiant les informations. Mais ils n'ont rien compris. Pour de jeunes enfants, c'est inadapté.»

E6: « Tout ce qui a été fait a plu... tout ce que je demandais, on faisait tout pour que je puisse le faire, même si ce n'était pas vraiment en rapport avec la Maison du Volcan, par exemple des visites de fermes. J'ai été très content des intervenants.» 
C8: «En classe volcan, j'ai ressenti un certain autoritarisme qui nous a mis mal à l'aise. Pour le questionnaire que les élèves ont eu à remplir à la fin de la visite, nous n'avons pas été consultés pour savoir si le contenu nous satisfaisait ou pas.»

$\mathrm{C} 11$ : «J'ai assisté à une présentation des classes de terrain mais je pense qu'ils veulent aller trop loin et que ça ne va pas pour des quatrièmes, et même à la limite pour des élèves de lycée. Par exemple, j’ai entendu le responsable nous présenter des phénomènes géologiques en entrant dans des explications, mais je voulais lui dire d'arrêter parce que pour des quatrièmes ça leur passerait au-dessus de la tête.»

L'argumentaire développé autour de l'âge des élèves est contradictoire avec le discours d'autres enseignants évoluant devant le même public scolaire. Reste le problème de la complexité: soit l'enseignant n'est pas parvenu à rendre l'offre pédagogique accessible par la mise en place d'une situation didactique pertinente de ce point de vue, ou bien il s'agit d'un manque de soutien pédagogique, en ce sens que la contribution des intervenants n'a pas eu lieu ou n'a pas donné les résultats escomptés.

Selon les enseignants de collège, il s'agit essentiellement d'un problème de concertation. L'encadrement pédagogique muséal n'est pas assuré à l'initiative de l'enseignant ou en collaboration avec lui. En fait, la mise au point d'un questionnaire et de fiches activité par le service pédagogique répondant de manière standardisée aux besoins des élèves ne constitue pas une adaptation suffisamment individualisée vis-à-vis des objectifs et des attentes de chaque classe.

Notons que les lycéens ne bénéficient pas d'un encadrement pédagogique proposé par le musée. Toutefois, leurs professeurs n'hésitent pas à formuler des critiques de fond sur l'apport de la pédagogie muséale dans le cadre de la géologie et du volcanisme.

L14: « Le niveau est nettement insuffisant en ce qui concerne la volcanologie... cela permet de rafraîchir la mémoire des élèves. Mais on attend plus de réalité!»

L17: «Par rapport au programme qu'on a à faire, le volcanisme est une toute petite part. »

Il est cohérent de relever ces observations au niveau du lycée et en filière scientifique. L'ambition des programmes dépasse en effet le niveau de contenu et de vulgarisation scientifique proposé par le musée dont l'approche reste en partie tous publics.

De façon constructive, certains enseignants, à tous les niveaux d'enseignement, précisent néanmoins que certains paramètres sont nécessaires à la réalisation effective des objectifs fixés : mettre en place un projet ou déterminer un parcours; disposer d'intervenants formés pour faire passer le message scientifique; élargir l'offre pédagogique par la collaboration avec des organismes scientifiques. 
E1: «Pour participer aux classes volcan, j'ai mis en place un projet qui développait les objectifs de compétences transversales, avec en priorité la maîtrise de la langue. »

E6: «Certains intervenants ont été formés, d'autres pas. Lorsque les enfants ne comprennent pas, l'intervenant ne modifie pas sa façon d'expliquer, et reformule de la même manière. Il y a un problème de langage de l'intervenant. Il ne sait pas que ça vient de sa façon de présenter les choses. Et il attend une réponse qui n’arrive pas... Il faudrait des partenariats avec des personnes compétentes...»

C10: «L'exposition permanente présente beaucoup de choses donc il faut cibler les informations par un questionnaire. Ce serait bien aussi de faire une sorte de parcours ludique pour que les élèves trouvent ça moins lourd.»

L14: «Ce que j'aimerais voir pour les lycées, ce serait l'association de balades sur le terrain. Il faudrait également une salle qui accueillerait des élèves en formation continue et où on reconstituerait, à partir de photographies prises sur le terrain, l'histoire de l'île... Il faudrait que les élèves puissent discuter avec des professionnels... qu'ils soient confrontés à la réalité, qu'ils voient le matériel avec lequel les scientifiques travaillent et qu'ils s'interrogent sur ce travail. »

Cette démarche rappelle la prééminence du rôle des enseignants pour faire en sorte que la visite scolaire se caractérise par un partenariat fructueux mais aussi l'influence de leurs perceptions ou représentations des apports muséaux (Eidelman et Peignoux, 1995; Le Marec, 1998).

Les enseignants confirment en tout cas qu'ils ne peuvent évoluer de manière autonome dans le cadre muséal: ils recourent nécessairement à des intervenants qualifiés et recherchent même un élargissement des partenariats. Au niveau de l'école primaire en particulier, on peut y voir une illustration du phénomène croissant de partage de la responsabilité pédagogique entre le maître et d'autres adultes. Le modèle «monopolistique » traditionnel de l'enseignement par le seul instituteur n'est plus opératoire, bien que certains s'en défendent.

Ceci a des répercussions évidentes sur le bilan des apprentissages que peuvent dresser les professeurs de leur expérience muséale. De fait, certains enseignants ne donnent pas d'appréciation explicite du niveau d'acquisitions de leurs élèves: ils éprouvent même des difficultés à s'exprimer sur ce point. Pour E2, les résultats sont peu concluants eu égard aux objectifs et aux attentes extrêmement modestes qu'il formule par ailleurs. De même, C9 et C11 insistent sur les possibilités «limitées» de leurs élèves ${ }^{5}$. Pour C8 et C12, la sortie apporte quelque

5. Transparaît alors une thèse «sociologique», en ce sens que l'on se limiterait à des apports en matière de découverte pour des enfants de milieu défavorisé, rejoignant ainsi certaines attentes observées en ZEP (voir par exemple Rochex, 1997). Le partenariat ZEP-Musée a cependant, parfois, donné des résultats encourageants (Duval, 1999). 
chose (mais on ne sait pas vraiment quoi) aux élèves, sachant que C8 axe ses propos sur l'apport décisif du terrain. Enfin les propos emphatiques de E1 et E4 semblent attester d'un bénéfice pédagogique important, peut-être plus significatif dans le domaine non cognitif.

\section{Développement des compétences comportementales et difficultés liées à l'abstraction des notions étudiées}

Plusieurs enseignants insistent en effet sur l'apport du musée à ce niveau: les élèves acquièrent davantage d'autonomie, de responsabilisation, de socialisation, d'ouverture... au lycée, L13, L17 et L18 mettent particulièrement en avant l'apport des classes volcan et de la sortie au plan de l'appréhension du concret, de la motivation, des échanges, de la conduite d'une démarche active, de l'éducation civique et de la socialisation.

E1: «On essaye de faire apparaître des compétences transversales, des liens entre les disciplines. Par exemple, la vie sociale: les élèves vivent une semaine entière ensemble...»

E4: « Ce qui est bien aussi, c'est que les enfants peuvent se déplacer et être en autonomie, qu'ils fassent le geste d'aller chercher l'information et de ne pas l'attendre... les élèves s'unissent beaucoup plus lorsqu'on fait un projet commun. »

L13: «La classe volcan était le moyen de faire de la géologie de terrain. Les élèves ont pu voir concrètement, poser des questions, faire preuve de curiosité et de motivation. Ils ont beaucoup échangé entre eux. Ils ont essayé d'avoir une démarche de chercheur, de comprendre entre eux, d'aller eux-mêmes compléter leurs données dans le musée. »

L17 : «Il y avait cette autonomie parce qu'ils pouvaient chercher eux-mêmes, se balader partout, gérer leur temps. C'est surtout le terrain mais aussi d'autres apports qui sont intéressants, comme la vie en communauté. Il n'y a pas que l'aspect scientifique. Il y a une vie de groupe qui permet de créer un dynamisme dans la classe. On a fait des progrès considérables. Des élèves me disaient aussi que c'était super parce qu'on a passé de bons moments et on a pu s'amuser mais en travaillant. Et j'ai été étonné par la qualité de leur exposé... ça a été positif, pour leur permettre de faire preuve d'autonomie au niveau de la recherche documentaire, et puis faire un effort dans la présentation orale et se lancer. Dans la classe, il y avait des élèves qui ne s'étaient jamais parlés et là, ça a ressoudé des liens autres que scolaires... et le contact avec l'enseignant est beaucoup mieux après. »

L18: «Non seulement ils ont appris beaucoup de choses parce que je les ai obligés à prendre des notes, mais ça a permis de créer une certaine ambiance dans la classe. Ca m’a énormément aidé pour la suite. Ils sont devenus plus coopératifs et ils écoutent davantage. Ils posent des questions. Je vois très bien les élèves qui aident les autres lorsqu'ils n'arrivent plus à marcher ou lorsqu'ils ne comprennent pas quelque chose. Ils échan- 
gent beaucoup plus entre eux. Ils essayent de comprendre, ensemble ils essayent d'expliquer les phénomènes. »

À l'école primaire, l'acquisition de notions simples, objectif pédagogique prioritaire, est soulignée par plusieurs enseignants (E3, E5, E6), donnant ainsi une appréciation plus explicite du niveau d'acquisitions des élèves et de l'impact muséal attendu.

E3: « Ils ont un petit bagage notionnel qui leur permet de parler du volcan.»

E5: «En fin de parcours ils ont acquis certaines notions très simples.»

E6: «Ce qu'ils ont tous acquis c'est que le volcan c'est leur histoire, c'est à eux... généralement, ils ont tous compris le fonctionnement du volcan, les appareils de mesure, tout ce qui gravite autour du volcan, d'où vient le magma et comment lîle s'est formée, d'où vient la vie... Pour la structure de la Terre, les élèves arrivent à émettre des hypothèses. Et lorsqu'on a fait une bonne étude des volcans, ils arrivent à comprendre facilement tout ce qui est faille, plaque, tectonique, etc. »

Cependant, il ressort du discours de certains professeurs que des limites existent au niveau des acquisitions cognitives. En effet, selon eux, les élèves ne comprennent pas les notions abstraites. Les élèves ne comprennent que les choses qu'ils peuvent voir, décrire, toucher (voir par exemple Laperrière-Tacussel, 1995; Mouniama, 1996). Dans ces conditions, l'impact pédagogique muséal observé sur les élèves sera sans doute nuancé, voire totalement remis en cause (C9).

E3: «Comprendre, peut-être pas encore mais savoir ce qu'est un volcan.»

E5: « Au niveau du français ils auront progressé un tout petit peu, et pourront décrire ce qu'est un volcan en éruption, ce qu'ils voient. Mais il restera quand même quelque chose d'abstrait, de très lointain dans leur imagination. »

E7: «Par contre, au niveau des représentations, il y a des enfants pour qui le côté mystérieux du volcan est toujours présent. »

C9: «Il y a la Maison du Volcan qu'ils peuvent aller voir, mais en $4^{\mathrm{e}}$, je ne vois pas trop l'intérêt. On n'attend pas énormément des élèves et ça viendrait en plus. »

C10: «Il faut préparer cette visite. On ne peut pas lâcher les élèves comme ça.»

L14: «Ils pensent connaître le volcan. Mais ils restent au descriptif. C'est le sommet de l'iceberg. Ils ne peuvent pas comprendre le fonctionnement du volcan. Ça demande trop 
d'imagination. Pour les TPE, sur les séismes par exemple, on peut les faire travailler à partir des données physiques, mais ils ne disposent pas suffisamment de connaissances pour pouvoir les exploiter et comprendre ce qui se passe en réalité à l'intérieur. »

L17: «L'analyse des documents, c'est là que les élèves ont le plus de mal parce que ce n'est pas très rigoureux. Ils ont du mal à faire des déductions. Ensuite, pour ce qui est de la restitution, il y a souvent des hors sujets parce qu'ils ont du mal à bien cerner le problème. C'est pour cela qu'à la Maison du Volcan, je leur disais qu'il ne fallait pas tout prendre, qu'il faut faire un tri. Et souvent ils ont du mal à trier les connaissances. »

L18: «Les élèves n'aiment pas écrire. Ils n’aiment pas le théorique. Par contre, lorsqu'on leur donne du concret, ils sont plus intéressés. Et c'est là dessus qu'ils rattrapent leur note qui, en général, est assez faible. Ils sont incapables de comprendre des choses trop abstraites. Ca ne les intéresse pas. Et si on fait des sorties sur le terrain, ce sera facilement acquis par les élèves; Par contre, en restant en classe, à part quelques petites notions, il n'y a pas grand-chose qui reste.»

Au-delà de cette opinion radicale et finalement peu explicite, on relève deux avis (C10 et C11) exprimant des incertitudes quant à l'efficacité pédagogique de la démarche conduite en milieu muséal. On remarque aussi que L17 et L18 nuancent leur évaluation sur certains points méthodologiques, remettant quelque peu en cause l'image d'excellence généralement dévolue aux élèves de $1^{\text {re }} \mathrm{S}$. Un enseignant, L14, va encore plus loin, et ne semble finalement pas tirer un bilan positif en termes d'acquisitions pour ses élèves dans le domaine de la géologie. Ces propos suscitent une interprétation en forme d'interrogation quant à l'impact supposé de la pédagogie muséale.

\section{Conclusion}

On comprend bien que la construction des apprentissages scientifiques est valorisée le cadre du musée, produisant des acquisitions parfois limitées, mais il semble surtout que la difficile maîtrise conceptuelle de l'objet d'analyse (les phénomènes géologiques) oriente l'impact pédagogique recherché vers une dimension essentiellement communicative et socialisante.

Les objectifs notionnels simples proposés par les programmes au niveau de l'école primaire sont atteints sachant qu'il s'agit de la priorité déclarée par les enseignants. Pour le collège, le seul discours de C8 révèle des objectifs, des attentes, des choix pédagogiques et des modalités d'évaluation correspondant à une image éclairante et positive de l'apport de la pédagogie muséale. En revanche, chez les autres professeurs, s'impose une perception relativement « défaitiste » de l'enseignement de la géologie et des possibilités d'apprentissage de leurs élèves. Dans la même perspective, le jugement de L14 sur la capacité de compréhension de ses élèves de 
$1^{\text {re }} \mathrm{S}$ peut paraître inquiétant. Finalement, l'apport des activités pédagogiques en milieu muséal est reconnu et validé par les enseignants bien que certains d'entre eux stigmatisent des difficultés d'apprentissages qui ne sont pas toujours imputables à un échec ou une inadaptation de la pédagogie muséale. La place de celle-ci reste en débat, en tant qu'atout pédagogique complémentaire, décisif ou «compensatoire» au regard des attentes des enseignants et des aptitudes supposées des élèves.

\section{BIBLIOGRAPHIE}

Allard, M. et Boucher, S. Le musée et l'école, Cahier du Québec, Hurtubise, 1991.

Allard, M. et Boucher, S. Éduquer au musée, un modèle théorique de pédagogie muséale, Cahier du Québec, Hurtubise, 1998.

BILLET, A. L'exposition scientifique interactive: manipuler pour comprendre. Paris: CNRS éditions, 1996.

BRUner, J.-S. Le développement de l'enfant: savoir dire, savoir faire. Paris: PUF, 1983.

Caillet, E. et Coppey, O. Le faire-savoir ou la délectation au musée, in E. Faublée (éd.), En sortant de l'école... musées et patrimoine, Hachette Education, 1992.

Cohen, C. (2000), Contribution à l'étude des relations entre l'école et le musée. Vers une formation de l'enfant visiteur. Thèse, Paris: Museum National d'Histoire Naturelle, 2000.

DE VECCHI, G. et GIORDAN, A. L'enseignement scientifique: comment faire pour que "ça marche"? Z'éditions, 1996.

Doise, W. et Mugny, G. Le développement social de l'intelligence. Paris: Interéditions, 1981.

Duval, D. Donner le meilleur à ceux qui ont le moins! Un exemple de partenariat école/commune/musée pour favoriser la réussite scolaire et développer la culture scientifique en ZEP, Argos, $n^{\circ} 23,1999$.

Eidelman, J. et Peignoux, J. Les enseignants de l'école primaire et la Cité des sciences: les conditions du partenariat école-musée, La Lettre de l’OCIM, n 37, 1995.

Giordan, A. Apprendre! Belin, 2e édition, 1998.

Girault, Y. Prise en compte des intérêts des élèves dans le cadre de l'appropriation des savoirs scientifiques dans les espaces muséaux. Rapport de recherche CNCRE, ministère de l'Éducation nationale, 2000.

Guichard, F. et J. Des idées pour apprendre. CDDP, 1997.

Guichard, J. et Martinand, J.-L. Médiatique des sciences. Paris: PUF, 1999.

Héritier-Augé, F. dir., Les musées de l'Éducation nationale. Mission d'étude et de réflexion. Rapport au Ministre de l'Éducation nationale, de la Recherche et de la technologie, 1991.

Jantzen, R. Forces, faiblesses et difficultés de l'interactivité en muséologie, in Dossier interactivité, p. 30$35,1995$.

LAPERRIÈRE-TACUSSEL, M. Le volcanisme, du cours moyen à l'IUFM, In Représentations et obstacles en géologie, Aster, n² 20, p. 76, 1995.

LAROUCHE, M.-C. L'évaluation des programmes éducatifs muséaux: le cas des lieux historiques au Québec. Thèse de doctorat en sciences de l'éducation, Université Paris V, 1999.

Le MAREC, J. Perceptions du musée chez les enseignants du primaire, in F. Buffet (éd.) Entre école et musée le partenariat culturel d'éducation. Lyon: PUL, 1998.

Mouniama C. Le volcanisme au CM2: des obstacles aux concepts. Mémoire professionnel PE2, IUFM de la Réunion, 1996.

Paquin, M. La visite scolaire au musée. Québec, Montréal: Presses interuniversitaires, 1998.

Piaget, J. La psychologie de l'enfant. Paris: PUF, (Que sais-je?), n³ 369, 1971.

Quagliozzi, A. et Cohen, C. Vers le partenariat école-musée: mobilisation enseignante et expertise des élèves, in Eidelman, J. et Van Praet, M. (dir.) La muséologie des sciences et ses publics, Regards croisés sur la Grande Galerie de l'évolution du Muséum national d'histoire naturelle. Paris: PUF (Éducation et formation), 2000.

ROCHEX J.-Y. Quand l'école «s'adapte» à la diversité sociale. Questions à la politique ZEP et à sa mise en œuvre, in Bourdon, J. \& THELOT, C. (dir) Éducation et formation, L'apport de la recherche aux politiques éducatives. Paris: CNRS éditions, 1997. 
SChiele B. et Koster E.H. La révolution de la muséologie des sciences. Presses universitaires de Lyon, Ed. Multimondes, 1998.

VAn Praet, M. et Poucet, B. Les musées, lieux de contre-éducation et de partenariat avec l'école. Éducation et pédagogie, $\mathrm{n}^{\circ}$ 16, 1992. 